
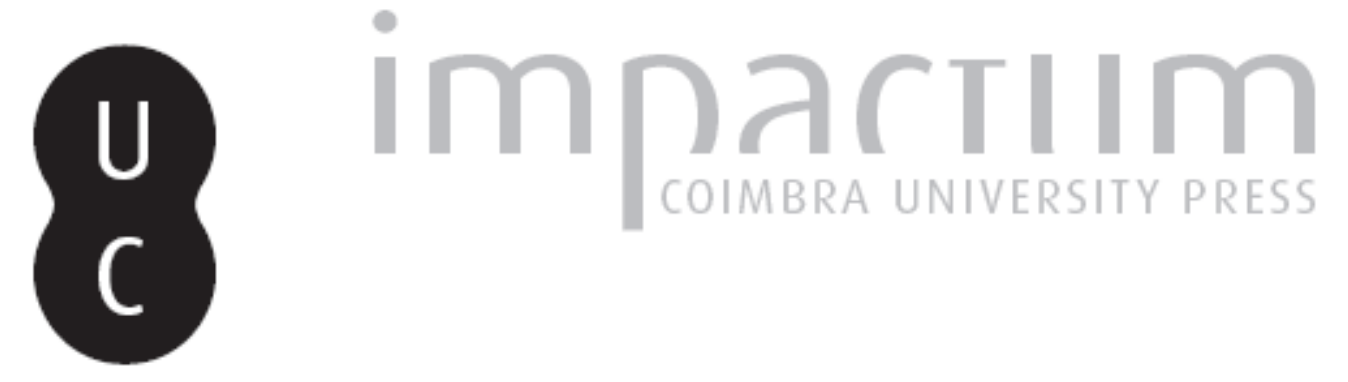

\title{
Academia, geopolítica das Humanidades digitais e pensamento crítico
}

\section{Autor(es): $\quad$ Pereira, Paulo Silva}

Publicado por: Centro de Literatura Portuguesa; Imprensa da Universidade de Coimbra

URL persistente:

URI:http://hdl.handle.net/10316.2/37788

DOI:

DOI:http://dx.doi.org/10.14195/2182-8830_3-1_7

Accessed : $\quad$ 26-Apr-2023 13:14:56

A navegação consulta e descarregamento dos títulos inseridos nas Bibliotecas Digitais UC Digitalis, UC Pombalina e UC Impactum, pressupõem a aceitação plena e sem reservas dos Termos e Condições de Uso destas Bibliotecas Digitais, disponíveis em https://digitalis.uc.pt/pt-pt/termos.

Conforme exposto nos referidos Termos e Condições de Uso, o descarregamento de títulos de acesso restrito requer uma licença válida de autorização devendo o utilizador aceder ao(s) documento(s) a partir de um endereço de IP da instituição detentora da supramencionada licença.

Ao utilizador é apenas permitido o descarregamento para uso pessoal, pelo que o emprego do(s) título(s) descarregado(s) para outro fim, designadamente comercial, carece de autorização do respetivo autor ou editor da obra.

Na medida em que todas as obras da UC Digitalis se encontram protegidas pelo Código do Direito de Autor e Direitos Conexos e demais legislação aplicável, toda a cópia, parcial ou total, deste documento, nos casos em que é legalmente admitida, deverá conter ou fazer-se acompanhar por este aviso.

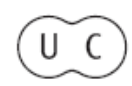




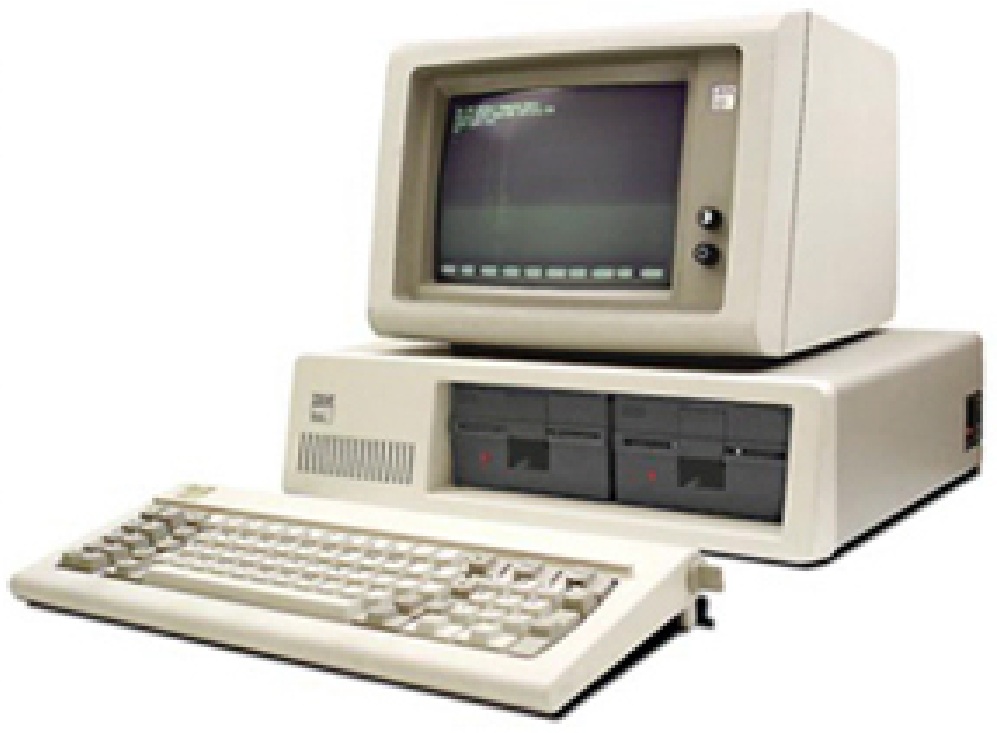

Vol. 3.1 (2015)

ISSN 2182-8830

'Artes, Média e Cultura Digital'

Paulo Silva Pereira e Pedro Serra (orgs.) 


\title{
Academia, Geopolítica das Humanidades Digitais e Pensamento Crítico Paulo Silva Pereira

\author{
CLP | Universidade de Coimbra
}

\begin{abstract}
Resumo
As mudanças que ocorreram no campo das tecnologias de informação e de comunicação nas últimas décadas tiveram impacto decisivo em vários setores da atividade humana, nomeadamente a nível de novas práticas criativas e de novos regimes de produção de conhecimento. Se é certo que durante algum tempo o esforço ia no sentido da integração de ferramentas informáticas no trabalho desenvolvido no campo das artes, humanidades e ciências sociais, mas numa lógica apenas de suporte técnico a tarefas academicamente consagradas, com a emergência do paradigma de Humanidades Digitais tornou-se mais visível o propósito de reinventar a cultura através da gramática dos novos meios e o crescente efeito de mediação da tecnologia digital no modo como decorre e se organiza a pesquisa ou se dá a conhecer os seus resultados finais. Palavras-chave: Academia; Humanidades Digitais; geopolítica e relações institucionais; diversidade linguística e cultural; pensamento crítico.
\end{abstract}

\section{Abstract}

The changes that occurred in the field of information and communication technologies in recent decades have had a significant impact on various fields of human activity, particularly on new creative practices and new regimes of knowledge production. It is true that for some time the effort was going towards the integration of computer tools in the fields of arts, humanities and social sciences, but only as technical support to established academic methods. However, with the emergence of the paradigm of Digital Humanities further transformations have become more visible, such as the purpose of reinventing culture through the grammar of new media, and the increasing mediating effect of digital technology on how research is organized and presents its findings to the public. Keywords: Academia; Digital Humanities; geopolitics and institutional context; linguistic and cultural diversity; critical thought.

\section{Humanidades digitais: o local e o global, o centro e a periferia}

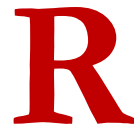

aro é o estudo que ao propor-se abordar o fenómeno das Humanidades Digitais não tenha início com uma reflexão, mais ou menos circunstanciada, sobre o alcance do conceito e não apresente uma proposta de cartografia do campo disciplinar que lhe corresponde. ${ }^{1}$ Descontando o que possa ter de lugar-comum da crítica a nível internacional, pois é

${ }^{1}$ Em Susan Schreibman et al., eds (2004), A. Burdick et al. (2012), D. M. Berry, ed. (2012) e M. K. Gold (2012) pode o leitor encontrar linhas de sistematização da históMATLIT 3.1 (2015): 111-140. ISSN 2182-8830

http://dx.doi.org/10.14195/2182-8830_3-1_7 
transversal a vários espaços geográfico-culturais ao longo dos últimos anos, o certo é que o exercício em si é sintomático da amplitude e complexidade desse diálogo entre áreas, tradicionais ou emergentes, que procuram tirar partido de inovações tecnológicas: literatura eletrónica, arquivos e bibliotecas digitais, observação georreferenciada de locais históricos, ferramentas Big data para potenciar a exploração de arquivos agregados, análise e utilização de redes sociais, para não alongarmos mais a lista. Acresce a tudo isto ainda a circunstância (benéfica) de se fazer acompanhar a dinâmica de criação e gestão de projetos com perfil digital de uma discussão, por vezes intensa em virtude de pontos de vista contrastantes, mas em todo o caso sempre profícua, sobre o impacto da tecnologia no processo de ensino/aprendizagem, na perceção que a sociedade tem do que se faz em contexto universitário e a nível da investigação científica propriamente dita. Não é difícil, regra geral, encontrar a linha divisória entre os que propõem uma tecnofilia triunfante, sempre dispostos a exaltar o efeito renovador (quando não, revolucionário) das últimas novidades, ainda quando os seus benefícios não foram bem avaliados, dos que procuram travar, a todo o custo, o avanço da tecnologia computacional no seio da Academia com receio de um irreversível efeito de descaraterização do que tem sido, nos últimos séculos, a matriz dominante da cultura humanística.

Para muitos, o advento deste novo paradigma de Humanidades Digitais, que não pode ser confundido de forma simplista com mera utilização de ferramentas informáticas aplicadas aos domínios linguístico, filológico e literário, pois essa começou há várias décadas e deu frutos generosos (bases de dados; tratamento automático de texto), foi lido como oportunidade de reinventar a própria missão universitária e de garantir um acréscimo de reconhecimento por parte da sociedade contemporânea. ${ }^{2}$ É hoje claro que, em função disso, as fronteiras do espaço universitário se tornaram mais porosas, pelo envolvimento de entidades (instituições culturais, empresas privadas ou agências de financiamento) e públicos mais numerosos e diversos em proje-

ria, dos princípios teóricos e das metodologias do campo das Humanidades Digitais. Uma parte relevante da reflexão tem vindo a público também sob a forma de manifestos, o que só por si é revelador do desejo de afirmação pública e do debate interno que decorreu nos últimos anos, como se pode comprovar pelos seguintes exemplos: A Digital Humanities Manifesto

(http:/ / manifesto.humanities.ucla.edu/2008/12/15/digital-humanities-manifesto/) The Digital Humanities Manifesto 2.0

(http:/ / manifesto.humanities.ucla.edu/2009/05/29/the-digital-humanities-manifesto20/) ou Manifeste des Digital humanities, apresentado no âmbito do THATCamp Paris 2010 (http:/ / tcp.hypotheses.org/318).

2 Para uma melhor compreensão do contexto especificamente português, é útil a consulta do ensaio de Daniel Alves (2014). Em "El mapa y el territorio. Una aproximación histórico-bibliográfica a la emergencia de las Humanidades Digitales en España” (2013), Antonio Rojas Castro oferece uma cartografia do fenómeno num país que, pela sua proximidade geográfico-cultural, poderá servir aqui como termo de comparação. 
tos colaborativos de criação e disseminação de conhecimento. Por outro lado, a utilização indiscriminada da etiqueta $H D$ para designar qualquer iniciativa da área das Humanidades ou das Ciências Sociais que se sirva, de forma mais intensa, de recursos digitais ou de tecnologia computacional é bem a prova da sua feição trendy e do lastro mítico de pioneirismo que parece conter a ponto de dar origem a formulações como the next big thing. ${ }^{3}$ No ensaio "What is "Digital Humanities," and why are they saying such terrible things about it?", que acaba por constituir (de forma involuntária, segundo o autor) a última peça de um tríptico dedicado à problematização de questões estruturantes deste campo e, convém dizê-lo, à refutação de várias críticas e objeções, Matthew Kirschenbaum tende a interpretar muito do que vem sucedendo como advento de um complexo e poderoso constructo discursivo. ${ }^{4}$ Outros estudiosos como Rita Raley, só para trazer um exemplo citado no texto, já tinham apontado esse sentido de construção discursiva, mas a forma acutilante como Kirschenbaum agora convoca a matéria é digna de nota, porque abre um espaço de discussão sobre o processo de formação do campo e sobre as densas relações de poder presentes e ativas no seu interior, descartando até certos lugares-comuns como o que tendia a ver a origem das Humanidades Digitais no sistema universitário norte-americano como ligada, acima de tudo, às instituições mais prestigiadas e financeiramente poderosas. Em todo o caso, é sobretudo a ideia da existência de práticas discursivas que formam (e transformam) sistematicamente os objetos de que falam que aqui

3 A fórmula rapidamente tornada célebre no contexto universitário e no espaço mediático foi pela primeira vez utilizada por William Pannapacker no contexto da Modern Language Association (MLA) Convention de 2009. O mesmo autor volta à questão com mais profundidade e aduzindo novos dados em "Digital Humanities Triumphant?” (http://dhdebates.gc.cuny.edu/debates/part/4).

${ }^{4}$ Este ensaio de M. Kirschenbaum foi inicialmente publicado na revista differences 25.1 (2014) como parte integrante do número especial In the Shadows of the Digital Humanities, coordenado por Ellen Rooney e Elizabeth Weed, mas encontra-se disponível para consulta no site do autor em

https:/ / mkirschenbaum.wordpress.com/2014/04/24/new-essay-what-is-digital-

humanities-and-why-are-they-saying-such-terrible-things-about-it/. Logo na parte inicial do ensaio, estabelece-se um breve, mas muito elucidativo, elenco de opiniões adversas quanto ao teor e efetiva validade das Humanidades Digitais: "Digital humanities is a nest of big data ideologues. Digital humanities digs MOOCs. Digital humanities is an artifact of the post-9/11 security and surveillance state (the NSA of the MLA). Like Johnny, digital humanities can't read. Digital humanities doesn't do theory. Digital humanities never historicizes. Digital humanities is complicit. Digital humanities is naive. Digital humanities is hollow huckster boosterism. Digital humanities is managerial. Digital humanities is the academic import of Silicon Valley solutionism (the term that is the shibboleth of bad-boy tech critic Evgeny Morozov). Digital humanities cannot abide critique. Digital humanities appeals to those in search of an oasis from the concerns of race, class, gender, and sexuality. Digital humanities does not inhale (easily the best line of the bunch). Digital humanities wears Google Glass. Digital humanities wears thick, thick glasses (guilty). Perhaps most damning of all: digital humanities is something separate from the rest of the humanities, and - this is the real secret — digital humanities wants it that way." 
importa recuperar, pois é aí que se encontra uma das razões mais fortes da assimetria entre o mainstream de matriz anglo-saxónica e outros horizontes geográfico-culturais. Para a configuração e limites do campo (do entendimento minimalista ao maximalista, capaz este último de congregar atividades muito díspares), para a discussão das questões epistemológicas e metodológicas mais densas, para a delimitação de traços distintivos do protótipo de humanista digital, concorrem vários atores e dinâmicas institucionais, mas a formação de consensos muitas vezes depende só de um núcleo restrito.

Quando se considera a história das Humanidades Digitais nas últimas décadas, torna-se inevitável reconhecer a forte preponderância da comunidade anglo-americana (fundamentalmente organizada em torno do eixo atlântico: Estados Unidos da América, Canadá, Reino Unido), na modelação deste campo disciplinar e na determinação de um conjunto de instituições internacionais com influência decisiva no tempo e no modo como se processou o seu desenvolvimento. Essa preponderância aparece representada de forma convincente na série de infográficos que Melissa Terras elaborou, em 2012, no âmbito do projeto Quantifying Digital Humanities, pois permite acompanhar a expansão do campo em termos de número de pessoas envolvidas, distribuição geográfica dos centros de pesquisa (na altura, 114 centros em 24 países, mas mais recentemente a autora apontava a existência de 195 centros em 27 países), ${ }^{5}$ quantificação de projetos e nível de financiamento obtido [Figura 1]. ${ }^{6}$ Para vários estudiosos, o modo como se processou este balanço (e, por conseguinte, o resultado final que assim se obteve) é fortemente redutor, pois tende a deixar de lado experiências de aplicação e uso de tecnologias, recursos e sistemas digitais em vários pontos do mundo que não respondem aos critérios definidos por essa grande narrativa. Como bem fazia notar Domenico Fiormonte em "Towards a cultural critique of the Digital Humanities", a estratégia delineada por Melissa Terras produziu uma flatlandia muito alargada donde emergem as figuras colossais do espaço anglo-saxónico. Fiormonte tem sido, de resto, uma das vozes mais críticas nos últimos anos quanto à redução drástica das genealogias múltiplas das Humanidades Digitais (e do seu caráter poligenético) e interessa-nos aqui muito pelo olhar desassombrado que projeta sobre os mecanismos que suportam esse ascendente de uma parte do mundo sobre a restante. Com lucidez (e alguma coragem, importa notar), enfrenta a delicada questão da composição geopolítica e linguísticocultural da disciplina e, como sua consequência, dos instrumentos utilizados e define problemas de duas ordens: por um lado, a constituição dos órgãos de

${ }^{5} \mathrm{~A}$ intervenção de Melissa Terras a que nos referimos teve lugar no âmbito da $D H$ Conference Benelux 2014 e tinha como principal propósito fazer um balanço do estado atual das pesquisas nesta área, como se pode ver em:

https:/ / www.youtube.com/watch?v=k_geG9cdIAs.

${ }^{6} \mathrm{O}$ núcleo fundamental dos resultados obtidos através desse levantamento conduzido pela investigadora encontra-se disponível para consulta em:

http://www.ucl.ac.uk/infostudies/melissa-terras/DigitalHumanitiesInfographic.pdf. 
governo das instituições que inspiram, gerem e regulam as estratégias e as metodologias de pesquisa (e consequente visibilidade dos resultados) e, por outro, as caraterísticas linguístico-culturais dos instrumentos: software produzido no mundo anglo-americano; problemas de ordem semiótico-cultural.

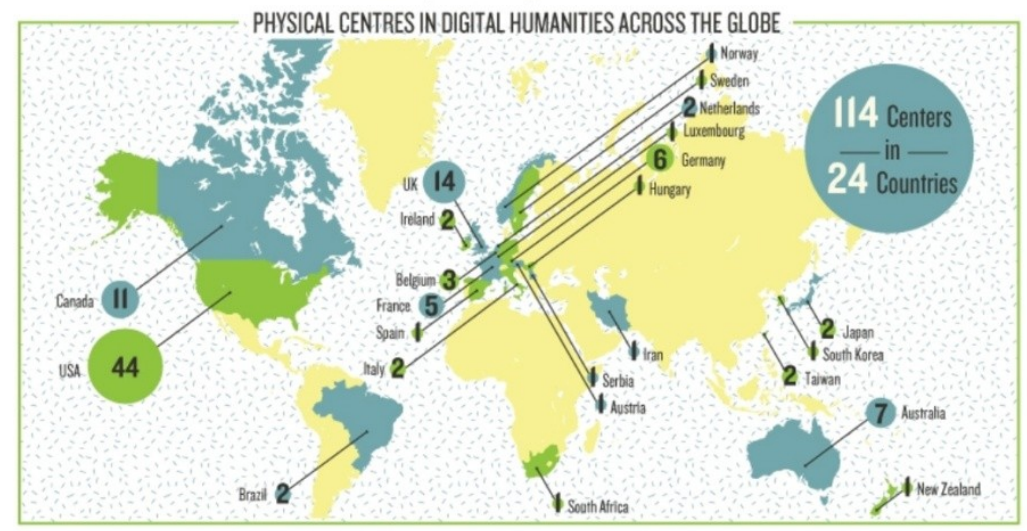

Figura 1. "Physical Centres in Digital Humanities Across the Globe." (C) 2012, Centre for Digital Humanities, University College London.

A excessiva concentração, que tem vigorado nas últimas décadas, em torno de um núcleo muito restrito de pessoas e de instituições, com claro predomínio da língua inglesa, deveria dar lugar a um novo modelo: uma comunidade de comunidades, que pudesse congregar o trabalho de humanistas digitais com suas várias sensibilidades. ${ }^{7}$ Se ninguém ousa pôr em causa a relevância da utilização de uma língua de trabalho comum como a língua inglesa, já o reduzido grau de diversidade cultural dos projetos devia merecer reflexão. ${ }^{8}$

${ }^{7}$ Foi recentemente criada uma nova organização, a Digital Research Infrastructure for the Arts and Humanities (DARIAH-EU), cuja principal missão consiste em estabelecer uma rede transnacional de cooperação, no espaço europeu, entre pessoas e instituições com vista ao desenvolvimento de ferramentas, metodologias e apoio de vária ordem para atividades de pesquisa na área das Humanidades Digitais. Além de permitir o mapeamento agregado de projetos e iniciativas de base europeia, o site da DARIAH-EU oferece ainda um elenco significativo de cursos e programas de ensino com planos de estudos digitais (https://dariah.eu/about.html). Outras associações e iniciativas têm vindo a sublinhar a importância da formação de conglomerados de escala mais significativa, como se percebe pelo trabalho realizado por parte de: Alliance of Digital Humanities Organizations (http://digitalhumanities.org), The European Association for Digital Humanities (EADH)); Association for Computers and the Humanities (ACH); Canadian Society for Digital Humanities / Société canadienne des humanités numériques (CSDH/SCHN); centerNet; Australasian Association for Digital Humanities (aaDH); Japanese Association for Digital Humanites (JADH).

${ }^{8}$ Segundo Domenico Fiormonte e Marin Dacos (este último num texto intitulado "La stratégie du sauna finlandais", 2013), entre as principais razões que têm contribuído para a reduzida visibilidade do trabalho realizado fora da corrente hegemónica em língua inglesa conta-se o acesso muito limitado aos órgãos de governo de instituições 
Demonstrando a pertinência em se discutir esta problemática, mais recentemente, Isabel Galina, na conferência que proferiu no congresso Digital Humanities (DH2013), "Is There Anybody Out There? Building a global Digital Humanities community", defendia (melhor: dava voz, porque o ponto é defendido por muitos) a necessidade de se promover um grau maior de representatividade linguística e geográfico-cultural na comunidade global, tendo por base o trabalho que vem realizando no projeto Red de Humanidades Digitales (RedHD), no México. ${ }^{9}$ Entre as questões mais decisivas que então formulava, encontra-se seguramente esta: "Behind this problem of defining Digital Humanities (what we are and what we do) there is an additional now ineludible problem "who is we?"”. Subjacente a esta questão está, como é evidente, o crescimento exponencial que se tem verificado a nível mundial e que traz consigo novos desafios e uma reconfiguração do tipo de relações com forte matriz hierárquica que tradicionalmente se estabelecem entre o centro e a periferia do sistema:

It is important that we understand that we sometimes unconsciously incorporate assumptions into our proposals and initiatives that do indeed

desta área a nível internacional (associações, eventos científicos ou direção de revistas). Tendo como tema central "Digital Diversity: Cultures, languages and methods", o congresso Digital Humanities 2012 que teve lugar na Universidade de Hamburgo, de 16 a 22 de julho de 2012, abriu amplo espaço à discussão destas matérias, desde a conferência de abertura "Dynamics and Diversity: Exploring European and Transnational Perspectives on Digital Humanities Research Infrastructures", a cargo de Claudine Moulin, diretora do Center for Digital Humanities da Universidade de Trier e autora de um relatório europeu sobre a questão (European Science Foundation, Research Infrastructures in the Digital Humanities, Science policy briefing 42, 2011, disponível em

http://www.esf.org/hosting-experts/scientific-review-groups/humanities-

hum/strategic-activities/research-infrastructures-in-the-humanities.html).

Outros investigadores têm procurado estudar toda uma série de implicações de ordem linguístico-cultural, como acontece com David Golumbia (2013), que refletiu sobre a relação entre Estudos Pós-coloniais, Humanidades Digitais e Política da Língua, ou como Amelia Sanz, em "Digital Humanities or Hypercolonial Studies", os efeitos que resultam de um certo tipo de estrutura organizacional, de gestão e de propriedade de arquivos e repositórios de larga dimensão. Para esta última, o desafio com que se confronta atualmente uma parte significativa da comunidade científica mundial merece atenta ponderação: "Bearing in mind the huge amount of data scholars will be able to handle in the near future, the Digital Humanities should not simply become the machinery to standardize knowledge as in a new positivist turn for the sake of the empire of hyper-reason as a universal structuring formation. Quite the contrary: more than ever, it is time for a new hermeneutical turn to locate different interdisciplinary, transcultural points of view on the Net." (2013)

9 Para um conhecimento mais aprofundado desse projeto, cf. http://www.humanidadesdigitales.net/. Citamos aqui o texto a partir da matriz que se encontra disponível em: http://humanidadesdigitales.net/blog/2013/07/19/is-thereanybody-out-there-building-a-global-digital-humanities-community/ Uma nova versão, revista e aumentada, do texto apresentado no congresso acima referido foi, entretanto, publicada na revista Literary and Linguistic Computing, sob o título de "Geographical and linguistic diversity in the Digital Humanities" (2014). 
affect inclusiveness or representation. We must be careful to avoid playing 'catch up' or initiatives that automatically assume that the objective is to "help" countries currently on the periphery to become just like the model DH centre. We can all learn and benefit from each other and collaboration should work in both directions. Methods that have worked effectively in one cultural setting may fail spectacularly in another (and vice versa) and certain reasoning of how things should work does not apply similarly to other frameworks. Models, surveys, truisms should be placed in context. Periphery countries can contribute by framing and stating more explicitly how and in what ways true collaboration can be achieved. I think that attitude is the keyword here. (Galina, 2013)

No dizer muito acertado de Matthew Gold, em Debates in the Digital Humanities, é um campo de tensões cujas fronteiras se encontram em permanente negociação e que gera efeitos próprios de um processo de crescimento: "a field in the midst of growing pains as its adherents expand from a small circle of like-minded scholars to a more heterogeneous set of practitioners who sometimes ask more disruptive questions". Em todo o caso, apesar da geometria variável deste campo e da proveniência geográfica muito diversa dos investigadores, é possível identificar um núcleo de princípios e de valores que estão na base de um sentimento de pertença a uma mesma comunidade: interdisciplinaridade; acesso aberto (open source); reconfiguração das normas de copyright e de propriedade intelectual por via da promoção de licenças alternativas (Creative Commons); trabalho colaborativo.

Quando se pensar em redigir, um dia, a história da génese e do desenvolvimento das relações entre os estudos literários, linguísticos ou culturais e a tecnologia digital ou, mais recentemente, a emergência das Humanidades Digitais no espaço transnacional de língua portuguesa, haverá por certo o cuidado de destacar o trabalho meritório de vários pioneiros das últimas três a quatro décadas, mas não será possível ignorar o 'momento' atual (triénio 13/15) como ponto fulcral da construção de um discurso legitimador e da consolidação de uma rede institucional-académica. Nunca antes se tinha visto uma mobilização tão forte de investigadores, organizações e centros de pesquisa em torno das relações entre Humanidades e Ciências ou de diálogos interdisciplinares cada vez mais desafiantes.

Ainda que sem a preocupação de se estabelecer uma lista exaustiva de eventos que dão conta desse interesse significativo em torno do campo das Humanidades Digitais, poderíamos aqui recordar: o DíaHD 2013, ${ }^{10}$ os congressos organizados pelo GRISO, da Universidade de Navarra, ${ }^{11}$ e pela

10 Día de las Humanidades Digitales 2013. http:/ / dhd2013.filos.unam.mx/

11 Humanidades digitales: visibilidad y difusión de la investigación. Grupo de Investigación Siglo de Oro (GRISO). Pamplona (23 e 24 de maio 2013).

http://www.unav.edu/congreso/humanidades-digitales/ 
HDH: Humanidades Digitales Hispánicas, na Universidade da Coruña, ${ }^{12}$ a formação da AAHD: Asociación Argentina de Humanidades Digitales, ${ }^{13}$ AHDig: Associação das Humanidades Digitais, ${ }^{14}$ a realização do Seminario de Humanidades Digitales de la Universidad Nacional Autónoma de México, do Ciclo de Humanidades Digitales na Universidade de Salamanca, ${ }^{15}$ do I Seminário em Humanidades Digitais na Universidade de S. Paulo (2013), ${ }^{16}$ das Jornadas de Ciencias Sociales y Humanidades Digitales de la Universidad de Granada, ${ }^{17}$ a publicação em 2014 do Arquivo Digital da PO.EX: Arquivo Digital da Literatura Experimental Portuguesa, ${ }^{18}$ a organização do Colóquio Internacional Estudos Literários Digitais, ${ }^{19}$ na Universidade de Coimbra, do Congresso de Humanidades Digitais em Portugal: construir pontes e quebrar barreiras na era digital, ${ }^{20}$ na Universidade Nova de Lisboa.

É de assinalar que a passagem de uma fase de estruturação atomística (investigadores a trabalhar de forma isolada ou em pequenos grupos) a uma fase de convergência e de consolidação do campo, que trará não só um efeito de potenciação do resultado final da pesquisa, por via da partilha de experiências e de ferramentas, mas uma visibilidade acrescida que não deixará de ter consequências a nível das instituições académicas e das condições de financiamento de projetos. Importa notar que estamos perante um universo de pesquisadores que têm em comum o interesse pelo digital, mas sobretudo a condição de falantes da língua portuguesa, replicando o que tem acontecido, por exemplo, no espaço universitário transnacional de língua espanhola. Aliás, podemos reconhecer fenómenos muito curiosos de simbiose entre pesquisadores das áreas linguístico-culturais do Português e do Espanhol, como procuraram mostrar as responsáveis pelo projeto MapaHD, Élika Ortega e Silvia Gutiérrez [Figura 2], ${ }^{21}$ ou o projeto Atlas de Ciencias Sociales y

12 Humanidades Digitales: desafíos, logros y perspectivas de futuro. Humanidades Digitales Hispánicas. Sociedad Internacional. A Coruña (9-12 de julho 2013) http:/ / hdh2013.humanidadesdigitales.org/

13 AAHD: Asociación Argentina de Humanidades Digitales. http:/ / aahd.com.ar/

14 AHDig: Associação das Humanidades Digitais. https://ahdig.wordpress.com/

15 Ciclo de Humanidades Digitales, Universidade de Salamanca (3 de outubro a 12 de dezembro de 2013). http://revistacaracteres.net/2013/11/ciclo-de-humanidadesdigitales-en-la-universidad-de-salamanca-cuarta-sesion/

16 I Seminário em Humanidades Digitais, Universidade de S. Paulo (2013). https://seminariohumanidadesdigitais.wordpress.com/

17 Jornadas de Ciencias Sociales y Humanidades Digitales, Universidad de Granada. Granada (16 e 17 de dezembro 2013). http://grinugr.org/noticias-de-eventos/i-jornadas-deciencias-sociales-y-humanidades-digitales-de-la-universidad-de-granada/

18 Arquivo Digital da PO.EX: Arquivo Digital da Literatura Experimental Portuguesa. https://po-ex.net/

19 Colóquio Internacional Estudos Literários Digitais, Universidade de Coimbra. https: / / eld2015.wordpress.com/about/

20 Congresso de Humanidades Digitais em Portugal: construir pontes e quebrar barreiras na era digital, Universidade Nova de Lisboa. https:/ / congressohdpt.wordpress.com/

${ }^{21}$ Cf. http://mapahd.org/el-mapa/. Para uma análise detalhada dos objetivos e da configuração do projeto, assim como dos resultados finais obtidos, pode consultar-se 
Humanidades Digitales (\#AtlasCSHD), ${ }^{22}$ desenvolvido pelo centro de investigação GrinUGR da Universidade de Granada, em Espanha, que pretende dar visibilidade acrescida a uma comunidade de académicos digitais com este perfil específico, mediante a identificação e geolocalização num mapa de investigadores, projetos, centros ou outro tipo de recursos.

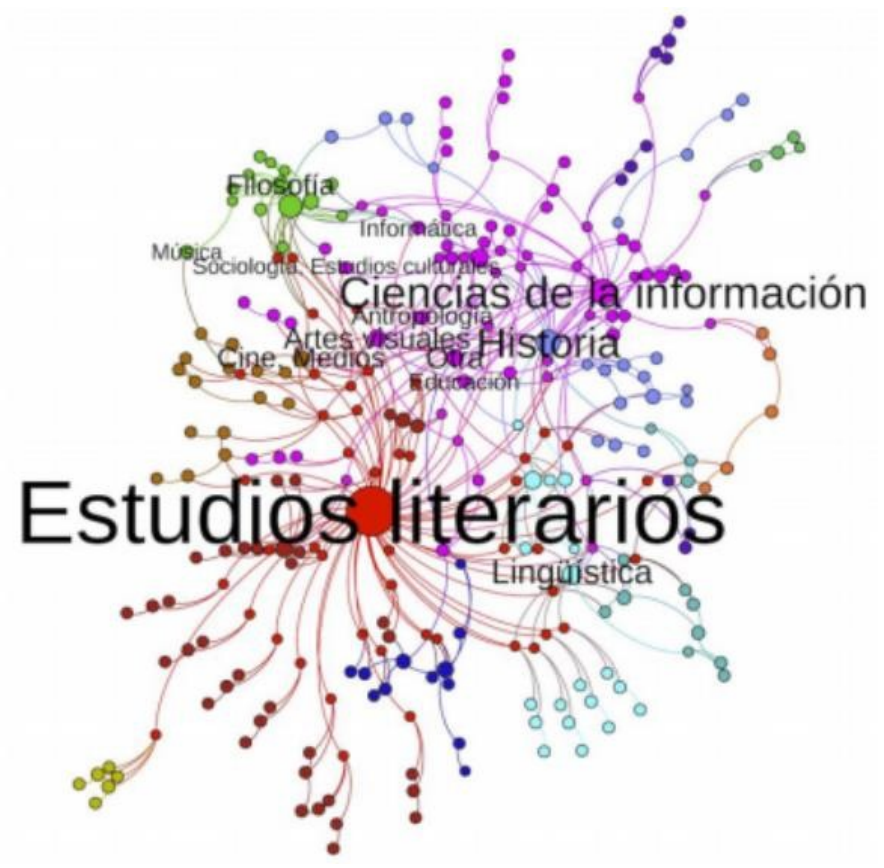

Figura 2. Mapa HD. (C) 2014, Élika Ortega e Silvia Gutiérrez.

\section{Building \& Knowing}

Um dos debates que mais contribuiu para a definição da differentia specifica deste novo paradigma das Humanidades Digitais foi o que se travou entre Stephen Ramsay, Geoffrey Rockwell e outros investigadores que tendem a reforçar, de modo vigoroso, uma "epistemologia materialista" que aposta sobretudo na componente de construção (building) dos artefactos digitais, e outros intervenientes, como é o caso de Alan Liu, que reconhecem importância vital à função crítica (traduzível aqui pela fórmula knowing) que acompanha desde sempre o perfil institucional e a base das formações disciplinares

com proveito o artigo "MapaHD. Una explotación de las Humanidades Digitales en español y portugués" que E. Ortega e S. Gutérrez publicaram no volume organizado por E. Romero Frías e María Sánchez González (2014).

22 Cf. http://grinugr.org/proyectos_internos/atlas-de-ciencias-sociales-yhumanidades-digitales/ 
da área das Humanidades. ${ }^{23}$ A face mais viva e visível da polémica teve como palco o congresso da MLA em 2011 e como mola propulsora uma intervenção de Ramsay intitulada “Who's In and Who's Out" com certo pendor radical, por se considerar que sem o domínio apurado de certas competências técnicas (saber construir ferramentas e sistemas ou utilizar código de programação, a título de exemplo) não seria possível fazer parte deste campo de pesquisa, mas a questão de fundo continua bem presente e atual. ${ }^{24}$ Que o modelo de knowing que passou a vigorar (e que se tornou quase irreversível) já não prescinde de uma componente mais robustecida de building ou making é algo que não parece muito difícil de aceitar, até mesmo por parte dos mais céticos, mas conceber um cenário de franca supremacia do último elemento sobre o primeiro pode ser ousado.

Seria tão relevante assim a figura de um intelectual tecnologicamente emancipado, capaz de realizar, por si só, tarefas de elevado grau de complexidade técnica, como faz crer Ramsay, ou deveria a sua missão passar, em primeira instância, por saber formular as questões decisivas em estreita articulação (e não em direta competição, porque essa estaria seguramente perdida) com especialistas?25 A opinião de Alan Liu sobre a resistência em teorizar o alcance cultural mais profundo da disciplina ainda continua a colher no momento atual: "the digital humanities are not ready to take up their full responsability [para revigorar as Humanidades] because the field does not yet possess an adequate critical awareness of the larger social, economic, and cultural issues at stake" (2011a: 11). Independentemente dos protagonistas em jogo em cada momento, a questão sempre volta à ribalta das discussões no interior do campo, o que mostra a sua natureza nuclear e decisiva para o desenvolvimento futuro. Quando desafiado a pronunciar-se sobre um ponto controverso, o de saber se existe uma 'agenda intelectual' específica (e definidora) de Humanidades Digitais ou se são apenas uma 'infraestrutura', Willard McCarty não hesitava em sublinhar a condição subsidiária desta última, pois apesar da sua indiscutível utilidade e da estabilidade institucional que garantiu, está longe de preencher as atribuições próprias do ofício de humanista: "Of course the digital humanities has to do with infrastructure; it has proved itself eminently capable of providing it, not just in the Text Encoding Initiative brilliantly but in numerous other projects as well. That's good, a fine and

23 Outros investigadores (Presner, 2012; Honn, 2013; Lothian e Phillips, 2013) têm vindo a salientar a relevância de uma abordagem crítica, mais densamente crítica do que habitualmente acontece, em articulação com o campo das ciências sociais e com domínios até agora marginais.

$24 \mathrm{O}$ fundamental da tese defendida por Ramsay encontra-se nos seus textos "Who's In and Who's Out" e "On building” (Ramsay, 2011a e 2011b).

25 Para uma abordagem das genealogias múltiplas das Humanidades Digitais, insistindo na tese de que as atuais concetualizações do campo derivam em grande medida da área de bumanities computing, mas incluem também outros contributos muito relevantes da análise de novos média, da teoria pós-colonial, dos estudos sobre a tecnologia e de tantos outros domínios, ver o estudo de Adeline Koh (2014). 
noble public service, but it's far from enough in the context of scholarship" (McCarty, 2012).

Desenvolvimentos recentes de tecnologias computacionais têm levado a que a ênfase posta, durante certa fase, nos processos de representação de fontes primárias esteja a ser agora orientada para a construção de instrumentos com vista à elaboração de conteúdo informativo, o que faz com que a marca diferencial não seja apenas a utilização de ferramentas tecnológicas, mas a procura de novos modelos interpretativos e de novos paradigmas no que toca à compreensão da cultura. Com esta transformação tem sido possível, como já sublinharam vários investigadores, potenciar uma das dimensões mais desafiantes da tecnologia computacional: a capacidade de analisar de forma mais fina os processos ainda antes dos produtos finais que deles resultam. Todd Presner e demais responsáveis pelo Digital Humanities Manifesto 2.0 destacavam já a relevância dessa mudança de rumo que resultou da implementação do princípio de conhecimento iterativo, das colaborações mobilizadas e das redes de investigação: "Process is the new god; not product. Anything that stands in the way of the perpetual mash-up and remix stands in the way of the digital revolution. [...] It honors the quality of results; but it also honors the steps by means of which results are obtained as a form of publication of comparable value" ( Schnapp e Presner, 2009).

A multiplicação de projetos de edição eletrónica e de construção de arquivo digital, numa zona de convergência entre mutações tecnológicas e teoréticas assinaláveis, trouxe para primeiro plano o debate em torno das formas de textualidade próprias do meio digital e dos reflexos que este projeta sobre o entendimento da arquitetura do livro da era pré-digital. Desde logo, a tarefa de codificação dos fragmentos textuais, por mais que se queira fazer passar a ideia de processo técnico e objetivo, nunca dispensa a componente analítica e reflexiva, pelo que se assume como método de edição que tem subjacente a perceção do que é um texto, o seu modo de instanciação física, os aspetos mais relevantes da representação impressa - incluindo a dinâmica relacional criada pela disposição gráfica - e o modo como se concretiza a sua passagem para o meio digital. ${ }^{26}$ Neste sentido, vale a pena lembrar a reflexão desenvolvida por Willard McCarty em torno do conceito de modelling (data modelling): para ele, o modelo designa "a representation of something for purposes of study, or a design for realizing something new", ao passo que modelling remeteria para "the heuristic process of constructing and manipulating models" (McCarty, 2004: 24). Em termos práticos, o humanista digital constrói um modelo do seu objeto de conhecimento e desenvolve estratégias para conhecê-lo melhor; como tal, o modelo não se apresenta apenas como réplica ou simplificação da realidade, mas também como meio para explorá-la. Em Portugal, contamos já com uma fileira de projetos de

${ }^{26}$ Para uma problematização mais aprofundada de alguns destes pontos, cf. Pereira, 2015. 
arquivo digital que trouxeram consigo uma reflexão aprofundada (mensurável em número de textos publicados) sobre a dinâmica de representação do conhecimento em contexto digital (o que significa 'construir' com ferramentas digitais). ${ }^{27}$

\section{Universidade e novas formas de construção do conbecimento}

Que os paradigmas da pesquisa humanística sofreram uma mudança assinalável por via da incorporação de métodos quantitativos, já utilizados com grande insistência nas ciências naturais e sociais, ou de modelos e ferramentas computacionais, parece indesmentível, mas um diagnóstico cuidadoso levaria a reconhecer que as mudanças em curso na Academia são, regra geral, muito mais de ordem cultural do que propriamente tecnológica. Se se considerar, a título de exemplo, o trabalho que vem sendo realizado no campo da preservação digital nas últimas décadas, depressa se verifica que o esforço de construção (e posterior disponibilização) de corpora linguísticos e de bases de dados ou da digitalização em larga escala de coleções de bibliotecas e de museus, que dominou a fase inicial de utilização de tecnologia computacional, se faz acompanhar agora de uma atenção crescente à complexidade e especificidade dos meios, ao contexto histórico, à profundidade analítica e à interpretação, à curadoria e socialização dos recursos digitais. ${ }^{28}$

Há cerca de dez anos, numa comunicação apresentada ao congresso The Face of Text: Computer-Assisted Text Analysis in the Humanities sob o titulo de "Forms of Attention: Digital Humanities Beyond Representation", John Unsworth reconhecia já uma tendência que viria a mostrar-se decisiva neste campo disciplinar: "We are arriving at a moment when the form of the attention that we pay to primary source materials is shifting from digitizing to analyzing, from artifacts to aggregates, and from representation to abstraction". Em resultado do aumento significativo de bibliotecas e de arquivos digitais, sobretudo nalguns contextos geográfico-culturais, e do volume sem

27 Importa destacar, a este nível, dois casos relevantes: o cluster de projetos coordenados por Rita Marquilhas, do Centro de Linguística da Universidade de Lisboa: Projeto CARDS. Cartas Desconhecidas; P.S., Post Scriptum. Arquivo Digital de Escrita Quotidiana em Portugal e Espanha na Época Moderna, Projeto FLY. Cartas Esquecidas Disponível em: http:/ / cards-fly.clul.ul.pt/ e o projeto do Arquivo Digital do Livro do Desassossego, coordenado por Manuel Portela (para uma apresentação geral do projeto, cf. Portela, 2013b).

${ }^{28}$ Por uma questão de economia de espaço, mas também por se tratar de um domínio com configuração própria no âmbito internacional, na medida em que tem estado ancorado ao longo dos últimos anos numa rede relativamente autónoma de eventos científicos e de canais de difusão do conhecimento (revistas, meios eletrónicos...), não faremos aqui referência pormenorizada à Linguística Computacional. $\mathrm{O}$ nosso propósito fundamental passa por dar conta de um conjunto alargado de fenómenos que esteve (ou está) na origem de amplas zonas de interseção interdisciplinar entre humanidades, ciências sociais e horizonte digital. 
precedentes de informação que passou a estar disponível em condições de acesso muito facilitado, tornou-se urgente pensar sobre o modo como se podia tirar amplo partido desse material: "We've spent a generation furiously building digital libraries, and I'm sure that we'll now be building tools to use in those libraries... I'm sure that the texts won't go away while we do our tool-building - but I'm also certain that our tools will put us into new relationships with our texts" (Unsworth, 2004). O desenvolvimento de novos procedimentos de pesquisa, produção e interpretação com base em ferramentas informáticas trouxe consigo outros desafios no que toca à relação hermenêutica que o investigador estabelece com os textos (e isto independentemente da leitura que se possa fazer dos benefícios ou danos implicados por essa alteração).

A necessidade de lidar com ambientes infossaturados e de elevada complexidade como são os ambientes digitais contemporâneos, seja pela quantidade de dados que movimentam, seja pela heterogeneidade de práticas que convocam, trouxe para primeiro plano o debate sobre a pertinência do recurso a tecnologias digitais para uma abordagem mais sofisticada, logo qualitativamente melhor e não apenas impressiva sob o ponto de vista quantitativo, dos fenómenos culturais.

Nesse sentido, um dos debates mais acesos que atravessa o vasto território das Humanidades, mas talvez com mais acuidade até o campo dos Estudos Literários, diz respeito à utilidade (e consequente legitimidade) do que se designa sob a forma de leitura computacional ou leitura humana assistida por computador. Para muitos especialistas, os modelos de pesquisa fortemente ancorados no digital podem minar uma certa ideia, que se foi consolidando ao longo de quase um século, de coerência disciplinar em torno da prática de close reading ou de eventuais avatares seus com idêntica ênfase na minuciosa análise do texto em si. Que seja essa a matriz única ou, pelo menos, dominante na estruturação deste campo de pesquisa é algo que se pode debater longamente, mas que não importa de modo direto para o tipo de abordagem que aqui se desenvolve. Contudo, e uma vez que a atitude de suspeição, quando não de receio profundo, existe de facto e já fez correr muita tinta, será útil considerar o fenómeno com mais atenção.

Desde logo, a emergência de um novo paradigma de construção do conhecimento não significa necessariamente um corte radical com práticas anteriores, pelo que onde muitos apenas veem um processo de substituição, em que algo sucede a algo de diferente, seria mais apropriado reconhecer um efeito de sincretismo. Bastaria pensar num caso sintomático, com evidentes repercussões no âmbito da pesquisa universitária, mas também num quadro mais alargado de utilizações: o da leitura digital. É certo que a crescente importância de modalidades de leitura com este perfil veio desafiar a hegemonia da literacia tradicionalmente associada ao impresso, que vigorou durante séculos, mas seria abusivo (pelo menos, nesta fase e com os conhecimentos de que dispomos) pensar que estamos perante um cenário de alte- 
ração drástica de condições, até porque resta ainda explorar múltiplas zonas de intersecção entre o digital e o impresso. Lidar de forma eficaz com o aumento exponencial da quantidade de informação disponível pressupõe outras competências que vão muito para além do padrão tradicional de leitura e interpretação dos dados de pesquisa.

Em Transferred Illusions. Digital Technology and the Forms of Print, Marilyn Deegan e Kathryn Sutherland, depois de reconhecerem que as mudanças tecnológicas não servem para responder apenas às necessidades e aos anseios do presente, mas podem interferir decisivamente no que viermos a ser e no que viermos a fazer no futuro, põem em evidência essa nova ansiedade que as formas emergentes do digital e a remediação eletrónica trouxeram consigo:

We can expect that the circuits and pathways that the brain fashions in our use of the Internet will differ from those woven by the reading brain. And herein lies the new anxiety: could the instantaneous access of information, the machine processing of text and the kind of visual stimuli and screen absorption that substitute for reading in the digital space jeopardize the reading brain's precious reflective functions? (173)

Independentemente da resposta que se possa dar a esta questão (e sobretudo quando dispusermos de mais resultados de pesquisa empírica), não custa reconhecer que a proliferação do digital não só permitiu o acesso a mais livros (ou fontes de informação), como está a mudar também a relação que com eles mantemos. ${ }^{29}$ Não por acaso, professores e investigadores do espaço académico internacional têm vindo, nos últimos tempos, a reclamar a instauração de um regime de slow scholarship, apontando os efeitos nefastos que o acréscimo de rapidez e que o volume de informação trouxeram para certos processos típicos de investigação no contexto das Humanidades. ${ }^{30}$

Em todo o caso, os pesquisadores sentiram a necessidade de pôr em prática novas modalidades de identificar e de utilizar padrões que pudessem emergir dos dados postos à disposição por via dessas campanhas intensivas de digitalização. Domínios como a macroanálise, a prospeção de dados (data mining), a leitura distante ou o desenvolvimento de técnicas de visualização têm já (e terão mais ainda num futuro próximo) um impacto significativo nas práticas académicas, mesmo contando com a atitude de reserva dos que consideram que com isso se enfraquece a relação dos elementos textuais com o contexto em que os mesmos ocorrem ou se despreza o efeito de ambiguida-

29 Para uma abordagem estimulante e muito consistente de várias questões ligadas a este novo horizonte de práticas de leitura, cf. Portela, 2013a.

30 Para uma adequada problematização de várias questões que dizem respeito a este regime designado sob a forma de slow scholarship no contexto universitário atual, é muito útil a consulta do ensaio "The Slow University: Inequality, Power and Alternatives” que Luke Martell publicou recentemente (2014). 
de. ${ }^{31}$ Tornou-se possível tirar amplo partido da dinâmica de agregação de dados, alargando consideravelmente a escala do nosso olhar sobre os fenómenos culturais, mas isso depressa suscitou um debate em torno das instâncias de validação dessas conclusões mais alargadas a que agora podemos chegar. Lembremos, a este propósito, o breve ensaio de Daniel Cohen e Frederick Gibbs "A conversation wih data: prospecting Victorian words and ideas" que, partindo de um estudo de caso, sublinha o proveito resultante da conjugação entre métodos tradicionais, que potenciam uma exegese mais apurada dos textos, e métodos computacionais, que estimulam abordagens de largo espectro:

Far from replacing existing intellectual foundations and research tactics, we see text mining as yet another tool for understanding the history of culture - without pretending to measure it quantitatively - a means complementary to how we already sift historical evidence. The best humanities work will come from synthesizing "data" from different domains; creative scholars will find ways to use text mining in concert with other cultural analytics.

Apesar da existência de vários projetos nesta área, é cedo ainda para se fazer um balanço sério quanto ao verdadeiro potencial de certas metodologias, mas a mera hipótese de combinar algoritmos e tecnologia computacional para gerir e analisar, de forma eficaz e rápida, grandes volumes de dados provenientes de fontes muito heterogéneas convertendo-os em conhecimento não deve ser rejeitada em nome de um qualquer preconceito. Sabemos que um dos pontos mais sensíveis que sempre aflora quando se procura avaliar e discutir a existência de um eventual dark side of the digital humanities ou quando estão de algum modo em causa manifestações de ceticismo em torno da utilização de ferramentas computacionais diz respeito ao perigo de exclusão (ou, pelo menos, de marginalização) do bumano, obliterando mesmo, segundo alguns, questões de género, raça, religião ou etnicidade e à proliferação de abordagens consideradas demasiado simplistas. ${ }^{32}$ Quem perfilha esse ponto de vista esquece, porém, que a capacidade de formular perguntas relativamente aos resultados obtidos, de cruzar esses mesmos resultados com informações recolhidas por outros meios tecnicamente menos sofisticados ou de análise de contexto não desapareceu do horizonte do trabalho intelectual. O que o novo realinhamento disciplinar (admitindo que a mudança de paradigma é irreversível) trouxe consigo de desafiante foi esta possibilidade de se pensar, de modo mais aprofundado, as decisivas mutações que estão a ocorrer em termos de práticas de leitura e escrita, os problemas de escala e a possibilidade de lidar com corpora cada vez mais vastos e uma reconfiguração do

31 Cf. Borgman, 2015a, 2015b; Crane, 2006.

32 Cf., a título de exemplo, Bailey, 2011; Koh e Risam, 2013; Terras, 2013. 
trabalho académico, mas sem partir do pressuposto de que existe, ou deva existir, uma metodologia digital monolítica. Por outro lado, estamos aqui perante um quadro de pesquisa de elevada complexidade ou que requer pensar soluções tecnológicas próprias, pelo que obriga com frequência a contar com a colaboração de especialistas de perfil diverso num ambiente genuinamente interdisciplinar.

Como advertia Northrop Frye, o futuro de qualquer área de conhecimento não se faz sem um adequado aproveitamento do progresso tecnológico e é natural que tal progresso tecnológico acabe por transformar o próprio objeto de estudo dessas mesmas áreas, ainda que em determinada fase prevaleça um sentido de incómodo (ou de perplexidade) face aos novos desafios:

three of the most seminal mechanical inventions ever devised, the alphabet, the printing press, and the book, have been in humanist hands for centuries. The prestige of humanists in the past came largely from the fact that they lived in a far more efficient technological world than most of their contemporaries. It is true that today they are sometimes confused about the new possibilities opening up in front of them, though hardly more so than the rest of the human race and some of them may also be put off by over-enthusiastic forecasting. (2006: 457)

Influenciados que estamos pela vertigem da revolução digital, ingenuamente pensamos que esta metodologia de trabalho é recente, mas a verdade é que a elaboração de concordâncias e índices de obras como forma de facilitar a consulta de material fez parte desde cedo da trajetória histórica dos métodos humanísticos ou, se quisermos avançar até uma época cronologicamente mais próxima de nós, projetos como o que o Padre Busa desenvolveu com o apoio da IBM, na década de 50 do séc. XX, tendo por base a obra de S. Tomás de Aquino não se afastam muito das tentativas atuais de conceber modelos eficazes de pesquisa e de triagem de informação.

Num trabalho intitulado "Quantitative analysis of culture using millions of digitized books", um grupo multidisciplinar de estudiosos procurou abordar, a partir de um corpus de textos que representaria aproximadamente $4 \%$ de todos os livros alguma vez publicados (500 biliões de palavras), os efeitos provocados pela dinâmica de digitalização em larga escala que projetos como o Google Books têm levado a cabo, estabelecendo uma análise quantitativa de tendências culturais no âmbito de áreas humanísticas como a lexicografia, a gramática evolutiva, a memória coletiva ou a história das inovações tecnológicas, entre outras. ${ }^{33}$ Para designar este domínio escolheram o termo 
Culturomics, que teria por base "the application of high-throughput data collection and analysis to the study of human culture". ${ }^{34}$

Mas tomemos para análise um caso específico de aplicação de ferramentas Big Data nos Estudos Literários. Em obra recente (publicada em 2013), sob o título de Macroanalysis. Digital Methods and Literary History, Matthew Jockers, na linha do modelo de distant reading que Franco Moretti teorizou e pôs em prática ${ }^{35}$ propõe uma abordagem historiográfica que não se contenta em analisar apenas o território formado pelo número relativamente pequeno de obras que continuou a merecer atenção editorial ao longo de décadas ou séculos após a publicação inicial ou que são consideradas como decisivas para o estabelecimento do cânone literário. Nesse sentido, como faz questão de notar o autor, a proposta visa demonstrar "how a new method of studying large collections of digital material can help us to understand and contextualize the individual works within those collections", mediante a identificação de ocorrências linguísticas e de padrões semânticos em largos repositórios da narrativa ficcional dos séculos XVIII e XIX, boa parte dela praticamente esquecida hoje em dia [Figura 3]. Deixo de lado objeções que têm surgido pontualmente sobre a utilização que o autor faz dos métodos estatísticos, mas destaco uma observação crítica pertinente e reveladora, apesar de todos os méritos que a obra encerra: por mais que se queira fazer passar a ideia de evidências tangíveis (logo, irrefutáveis) resultantes da aplicação de métodos quantitativos, o certo é que tudo decorre de uma série de assunções sobre a natureza da linguagem que condicionam o teor do resultado final, provando uma vez mais que até em casos de distant reading não se pode prescindir de uma dinâmica interpretativa. A escala de análise interfere não só a nível da quantidade de textos que são objeto de tratamento numa dada pesquisa, mas também na formulação de novas questões, pois abre caminho a uma compreensão mais sistemática das convenções que regem as práticas literárias num determinado período histórico-cultural e do modo como certas obras canónicas se destacam face a um cenário de estereotipia semântica e formal. Por outro lado, é ilusório pensar que tenhamos alcançado já um nível satisfatório em termos de cobertura, pois uma parte significativa dos projetos mais recentes tem por base um corpus em língua inglesa, incidindo, sobretudo, sobre o séc. XIX e épocas posteriores, pelo que a escassez de dados abertos em determinados contextos do espaço transnacional de língua portuguesa continua a ser um entrave sério à expansão de certos métodos de trabalho.

34 É possível compreender melhor o alcance inicial do projeto, desenvolvido no âmbito do The Cultural Observatory, em Harvard, se tivermos em conta a definição da missão que se propunha levar a cabo: "is working to enable the quantitative study of human culture across societies and across centuries. We do this in three ways: i) creating massive datasets relevant to human culture; ii) using these datasets to power wholly new types of analysis; iii) developing tools that enable researchers and the general public to query the data." (http://www.culturomics.org/cultural-observatory-atharvard)

35 Cf. Moretti, 2005 e 2013. 


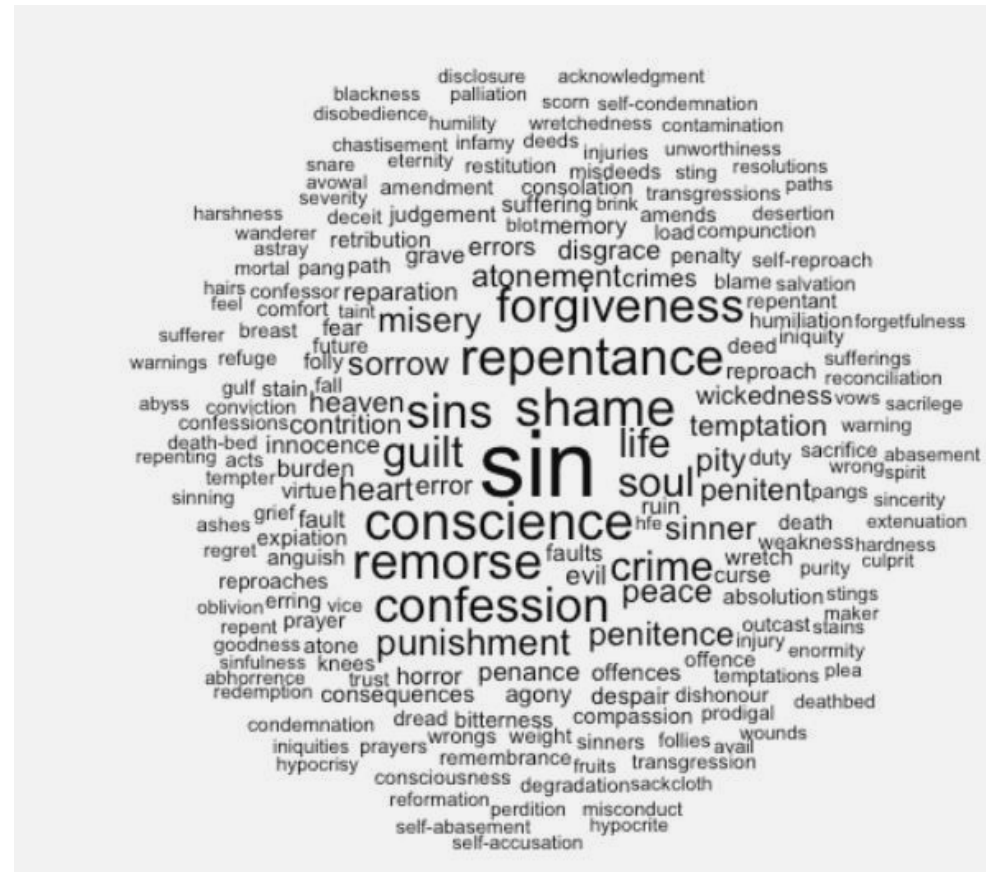

Figura 3. "Sin, Shame and Repentance". "500 Themes from a corpus of 19thCentury Fiction”, (c) 2013, Matthew L. Jockers.

Num ecossistema digital cada vez mais complexo com a proliferação de dados abertos, em larga escala e em contínua interação, é razoável pensar que se oferecem novas possibilidades no campo da investigação para as áreas das Humanidades e Ciências Sociais. Nesse sentido, a expansão do fenómeno Big Data não pode ser desligada de uma outra tendência forte das últimas décadas como é o Open Data, no sentido de livre disponibilização de dados online, sem os constrangimentos legais típicos do copyright e com uma natureza técnica que permite elevados níveis de utilização e de circulação de dados. ${ }^{36}$ Tendo em conta a complexidade crescente de tal estrutura formada por bases de

\footnotetext{
36 Segundo Viktor Mayer-Schönberger, professor e investigador do Oxford Internet Institute (OII), e Kenneth Cukier, editor de dados da revista The Economist, em obra publicada em 2013 sobre o fenómeno Big Data, nenhuma área da sociedade ficará imune aos seus efeitos, pelo que o meio universitário será também desafiado a utilizar e a desenvolver novas técnicas de gestão e de visualização de tais informações. $\mathrm{Na}$ verdade, aquele centro de investigação da Universidade de Oxford tem vindo a desenvolver um trabalho consistente neste campo, de que é exemplo o projeto Accessing and Using Big Data to Advance Social Science Knowledge, coordenado por Eric Meyer, que segundo se pode ler na memória descritiva disponível na página oficial (http://www.oii.ox.ac.uk/research/projects/?id=98) pretende analisar " "big data' from its public and private origins through open and closed pathways into the social sciences, and document and shape the ways it is being accessed and used to create new knowledge about the social world and the behavior of human beings."
} 
dados interconectados e abertos, de acesso público, estamos a caminhar cada vez mais no sentido de um paradigma que tem sido designado por alguns como Big-Linked-Open Data, com notáveis implicações sobre vários setores da atividade humana, incluindo o campo das Humanidades, nomeadamente a nível de protocolos de pesquisa e do surgimento de plataformas ou ferramentas Open Source em rede que permitem a recolha, análise e visualização de forma síncrona dos dados. Por outro lado, com as novas tendências educativas que têm vindo a tomar forma nos últimos anos, através de contextos de ensino/aprendizagem em rede (basta pensar no e-learning e nos MOOC's, cursos massivos online de forma aberta), apareceram outras oportunidades de aplicação de técnicas baseadas em Big Data que visam proporcionar um acompanhamento mais próximo dos estudantes, a tal ponto que já se fala de Educational Data Mining (ou Learning Analytics) como área disciplinar emergente. ${ }^{37}$

Em todo o caso, parece claro que o desafio mais relevante, num futuro próximo, passa por conceber a pesquisa como atividade que decorre, nas suas várias fases (identificação e seleção de fontes; aplicações práticas; publicação de resultados...), num ambiente colaborativo online e que, por isso, requer a constituição do que já foi designado por estudiosos como Gregory Crane, o fundador de Perseus Digital Library, como uma ciberinfraestrutura. ${ }^{38}$ Para trás parece ter ficado definitivamente a era do simples armazenamento e da conservação de documentos em suporte eletrónico, pois o que agora se procura alcançar é a totalidade do circuito que vai do acesso a conteúdos da herança artística e cultural ao seu estudo e posterior disseminação junto de um público cada vez mais alargado [Figura 4$].{ }^{39}$

37 Para uma primeira aproximação a esta problemática, revela-se útil a consulta do material disponível na página da International Educational Data Mining Society em http://www.educationaldatamining.org/. De acordo com os seus promotores, estamos perante "an emerging discipline, concerned with developing methods for exploring the unique and increasingly large-scale data that come from educational settings, and using those methods to better understand students, and the settings which they learn in."

38 Crane, Seales e Terras, 2009.

${ }^{39} \mathrm{Em}$ várias latitudes têm surgido consórcios, plataformas e redes de colaboração que apostam neste sentido de convergência interdisciplinar e de criação de novas infraestruturas para a pesquisa realizada na área das Humanidades e das Ciências Sociais. Vale a pena considerar aqui como ilustração desse modelo de espaço virtual de partilha científica os seguintes projetos: TAPoR 2.0 (http://www.tapor.ca/), NINES Nineteeth-century Scholarship Online (http://www.nines.org/), Interedition (http://www.interedition.eu/), TextGrid (https://textgrid.de/), CENDARI - Collaborative European Digital Archive Infrastructure (http://www.cendari.eu/), The Bamboo Project (http://www.bamboo-project.com/), Huma-Num (http://www.huma-num.fr/) ou, com alcance ainda mais abrangente, determinados consórcios formados nos últimos anos que podem ter papel decisivo, desde logo, na estandardização de procedimentos e tecnologias: o já referido $D A R I A H$ - Digital Research Infrastructure for the Arts and Humanities (https://www.dariah.eu/), CLARIN - Common Language Resources and Technology Infrastructure (http://clarin.eu/), NeDiMAH - Network for Digital Methods in the Arts and Humanities (http://www.nedimah.eu/). 


\section{CENDARI (Collaborative European Digital Archive Infrastructure)}

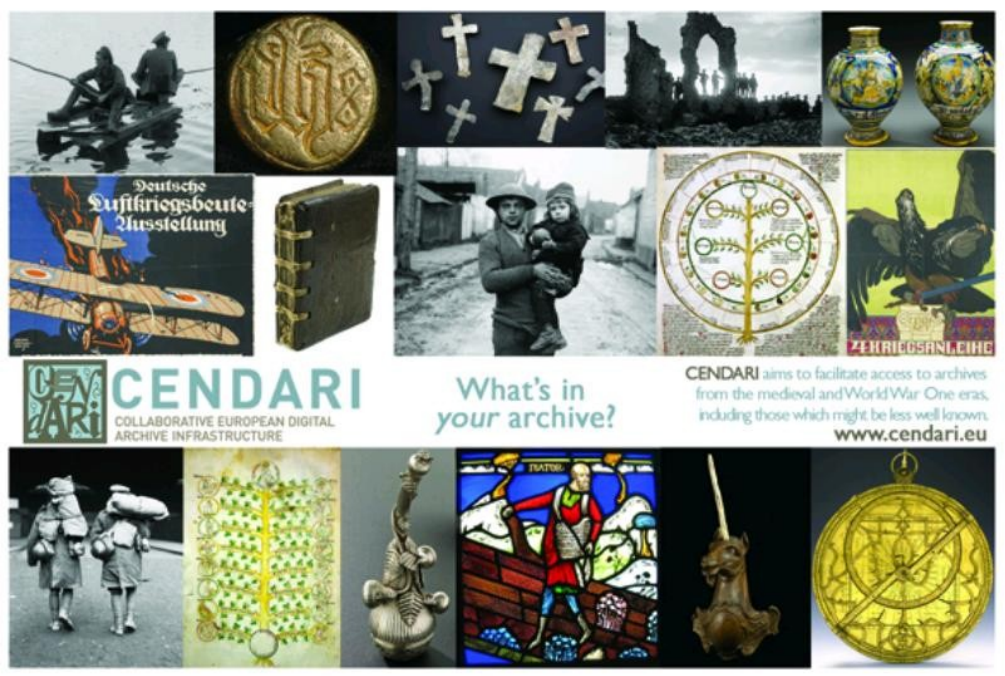

Figura 4. CENDARI. C 2015 Long Room Hub, Trinity College Dublin.

\section{Potenciar a disseminação e a transferência do conbecimento: o paradigma huma- nístico e os novos meios}

Um outro debate fortemente mobilizador que tem vindo a ganhar corpo no âmbito académico é o da influência das redes sociais e outras plataformas de social media nos procedimentos de pesquisa e na forma como se pretende dar a ver a uma audiência mais vasta os resultados práticos dos projetos. Num tempo em que a instituição universitária ela própria se vê obrigada, por pressão das atuais circunstâncias históricas (e isto não tem necessariamente um sentido trágico, porque sempre assim aconteceu), a reequacionar as suas funções e a renegociar o seu papel junto da sociedade, as tecnologias digitais estão a transformar o modo como entendemos e utilizamos a informação em circuitos que vão muito para além das instâncias tradicionalmente depositárias do saber. Dificilmente se pode conceber um renovado quadro de Humanidades no século XXI sem ter em conta a existência de mecanismos, mais densos e mais intensos, de transferência de saber oriundo do campo universitário. 40

40 Toda esta problemática da produção e disseminação do conhecimento é complexa e movente, uma vez que está diretamente vinculada a mudanças profundas de ordem tecnológica e de organização da sociedade contemporânea, mas é possível encontrar dados relevantes para uma reflexão mais aprofundada, incluindo sobre a necessidade de repensar formas tradicionais de avaliação ou sobre a abertura a novos modelos de participação na pesquisa científica, na obra conjunta de Antonio Lafuente, Andoni Alonso e Joaquín Rodríguez (2013). 
Mais do que em qualquer outra época histórica anterior e muito por força do perfil das novas tecnologias, hoje a ação do investigador raramente termina no momento da publicação dos resultados da sua pesquisa, pois este tem a responsabilidade de assegurar (ou, pelo menos, de contribuir para) um certo nível de impacto junto da comunidade científica e da sociedade em geral, seja pelo reforço da visibilidade do material produzido, seja pela acessibilidade mais facilitada. ${ }^{41} \mathrm{Da}$ publicação de trabalhos em plataformas digitais deriva, em muitos casos, um novo potencial de diálogo, quer com outros meios, quer com outros agentes (lectoescritores), que se traduz num certo número de interações em rede e leva à agregação de comentários ao texto original. Em lugar da matriz primordialmente substantiva que enformava a noção mais comum de conhecimento emerge agora um valor relacional que procura tirar partido da negociação entre perspetivas diversas.

Podemos certamente discutir a qualidade desse efeito de visibilidade acrescida e os modos por que se processa, uma vez que estamos longe de um cenário de consenso no que toca à utilização mais intensiva das redes sociais, do blogging no território das Humanidades, mas já ninguém ousa questionar o seu potencial. ${ }^{42}$ Partilham essas experiências, regra geral, o perfil de sistema aberto, não hierarquizado e sem um centro determinado, próximo do modelo rizomático proposto por Deleuze e Guattari, e nesse sentido representam um desafio à constituição e funcionamento do espaço académico tradicional, que sempre se regeu por valores doutra ordem.

Trata-se de uma aporia, na verdade, uma vez que a parte mais considerável da dinâmica das Humanidades Digitais teve início precisamente no seio da Universidade (e é por ela alimentada), mas o conjunto de pressupostos gerais que estão na sua base (abertura; partilha; forte sentido de colaboração; experimentação; risco; rasura de fronteiras disciplinares rígidas; novo entendimento da relação entre professor e estudante; reforço do impacto social da pesquisa; superação das dicotomias letras/ciências, teoria/prática, qualitativo/quantitativo; escala dos projetos de pesquisa) desafiam alguns pilares fundacionais do edifício académico que se foram consolidando ao longo do tempo, nomeadamente a partir do novo modelo de organização traçado por Wilhelm von Humboldt no século XIX. ${ }^{43}$

Independentemente do rumo que venha a ser seguido, há mudanças que interpelam de modo incisivo, no atual contexto, os agentes culturais que

$41 \mathrm{O}$ debate em torno da validade dos instrumentos utilizados para aferir o grau de impacto e de visibilidade da pesquisa científica tem sido particularmente intenso nos últimos anos, com várias vozes no contexto internacional a apostar na denúncia do que consideram ser modelos demasiados redutores. Tal não tem obstado, porém, a que apareçam cada vez mais ferramentas neste domínio: ImpactStory, ReaderMeter, ScienceCard, PLoS Impact Explorer, PaperCritic, Crowdometer, InCites, SciV al® Strata, SCImago Journal \& Country Rank, Scopus, Web of Science, Publish or Perish, Altmetrics, Google Scholar Citations.

${ }^{42}$ Cf. Escandell Montiel, 2012.

43 Cf. Röhrs, 1987. 
gravitam em torno do que tem sido considerado como Humanidades e Ciências Sociais. Uma dessas mudanças diz respeito à crescente necessidade de "produção de presença" (expressão que aqui tomo de empréstimo de Gumbrecht ${ }^{44}$ ) no meio digital, até como derivação de uma lógica avaliativa do desempenho para efeitos de promoção académica que tem em conta o grau de reconhecimento da figura do professor ou pesquisador no seio da comunidade científica e doutras esferas sociais.

O desafio maior consistirá em saber definir com lucidez a linha que separa a dimensão propriamente funcional, que se traduz numa série de ferramentas que estão ao serviço de uma estratégia legítima de publicitação do trabalho realizado em contexto científico, de um outro processo: o de espetacularização da vida universitária, que faz com que seja privilegiada a aparição em palcos sociais de relativa ou acentuada visibilidade em detrimento de uma prática de pesquisa mais sólida. Há um custo, em termos de tempo e de dedicação, nesse processo de construção de presença em contextos digitais que deve ser confrontado com o resultado final que o trabalho intelectual alcança por essas outras vias que até há poucas décadas não existiam ou tinham uma representação muito circunscrita.

Desde logo, é forçoso reconhecer que a nova forma de relacionamento dos seres humanos com o tempo (e com a História) que identifica a nossa contemporaneidade, com presença marcada de um forte efeito de aceleração, parece concorrer para a progressiva erosão de um modelo de atividade científica que se pautava pela profundidade e não pela busca obsessiva de um efeito de novidade (ainda quando só aparente). Nesse sentido, é legítimo questionar os fundamentos que estão na base da cultura de celebridades, num mercado do nome e da fama, que é transversal a toda a sociedade e que tem levado, no terreno intelectual, a situações de valorização (mediática) da figura do autor/investigador, num mundo globalizado, em detrimento da própria obra. O protótipo do intelectual ascético parecia já completamente ultrapassado em função da emergência da figura do investigador e intelectual nómada, em permanente trânsito entre eventos científicos em diversas partes do mundo, como satiricamente mostrou David Lodge no romance Small World, mas o cenário tornou-se mais complexo com a adição de novas valências como a construção e a aplicação de ferramentas digitais, o trabalho colaborativo em laboratório no âmbito de um grupo multidisciplinar e não apenas na solidão do gabinete ou, para o caso vertente, uma vivência online e offline. ${ }^{45}$

Os círculos científicos e intelectuais competem agora com outras instâncias de legitimação cultural, de julgamento e de acreditação do valor, ou com outros mecanismos de consagração dos autores que estão para além do modelo tradicional da comunidade de pares como fonte de auto-legitimação. É de assinalar, a este respeito, a discussão em curso sobre outras práticas de

${ }^{44}$ Cf. Gumbrecht, 2004.

${ }^{45}$ Cf. Lodge, 1984. 
avaliação distintas do tradicional sistema de peer review como seria o caso do open review ou do blog-based peer review, como foi designado por Noah WardripFruin, que permitem alargar a base de avaliadores, trazendo competências que doutro modo seria difícil conseguir pela sua especificidade técnica e por terem um forte enraizamento fora da Academia, e estimulam a leitura e o comentário de textos já concluídos ou ainda em fase de elaboração. ${ }^{46} \mathrm{~A}$ experiência proposta por Wardrip-Fruin tendo por base o processo de elaboração de Expressive Processing: Digital Fictions, Computer Games, and Software Studies é digna de nota, na medida em que conjuga a intervenção típica de blind peer review, revisão crítica anónima a cargo de um reduzido número de especialistas sob a égide de uma prestigiada editora (MIT Press), com uma outra desenvolvida no seio da comunidade online do blogue Grand Text Auto, que permitiu recolher comentários de indivíduos com proveniência diversa (indústria, universidade, produção artística, público em geral) com conhecimento profundo da área de jogos de computador, numa base de abertura intelectual e de intensa abordagem interdisciplinar. ${ }^{47}$ Talvez o ponto que deva ser sublinhado é o da meta-discussão que se promove sobre a natureza do envolvimento de setores da sociedade que se encontram fora dos muros da Academia, com as suas vantagens e com as suas eventuais limitações, mas tendo sempre presente que este tipo de projeto permite consolidar redes de conhecimento mais amplas.

O propósito de disseminação do saber, no quadro atual e com os meios de que dispomos, deve vir acompanhado da preocupação em evitar a banalização resultante de um efeito de hipervisibilidade dos agentes culturais, ainda quando esta parece responder a um desejo mais forte de intervenção do sector intelectual na esfera pública, ou da apresentação de resultados cada vez mais ténues e efémeros em virtude da proliferação de publicações, de eventos e de cumprimento de metas bibliométricas. Não se pode dizer que haja relação direta entre o estar permanentemente em linha, construindo e alimentando perfis de utilizador, e a qualidade da investigação que se desenvolve e, muitas vezes, esta última não é senão prejudicada por uma tal pulsão, sobretudo quando se torna compulsiva. É todo o problema da comunicação científica que está em pano de fundo.

Fórmulas como sociedade digital, cultura digital ou Humanidades Digitais tenderão a perder sentido com o tempo, pois mais não são do que constructos produzidos num quadro de profunda transformação epistémica (bastaria pensar na alteração dos princípios que regem a organização do conhecimento, do mundo de trabalho, das relações interpessoais e da base de constituição da identidade individual e coletiva), mas que não deixam de ser também

46 Para um breve olhar sobre o perfil desta discussão, cf. a informação disponível em: http://blog.f1000research.com/2014/05/21/what-is-open-peer-review/.

47 Wardrip-Fruin descreve em pormenor as várias etapas por que foi passando a pesquisa e a redação do texto em: http://grandtextauto.org/2009/05/12/blog-basedpeer-review-four-surprises/. 
emanações do fascínio pelo tecnológico numa sociedade em mudança, com forte potencial mobilizador. Com o aumento exponencial do peso da vertente computacional em numerosos setores da atividade humana e com a crescente ubiquidade da tecnologia, não será difícil prever que um horizonte de digitalidade altamente massificada tornará fatalmente obsoleta aquela marca diferencial. Por outro lado, as possibilidades de inovação serão mais significativas, pois a tendência para emular no meio digital os modelos textuais que se consolidaram ao longo de séculos no analógico deixará de ser tão recorrente, fazendo aumentar a capacidade de relacionar informação por parte do produtor de conteúdos, do leitor e do próprio meio. Do ponto de vista estritamente académico, não é de descurar ainda o efeito que a mudança geracional irá provocar, num horizonte de dez ou quinze anos, no entendimento dos pressupostos e da configuração do campo das Humanidades, pois alguns dos mais ativos defensores do novo paradigma hoje estarão, por essa altura, numa fase de maturidade em termos de carreira profissional.

Graças à rapidez e à facilidade com que as tecnologias digitais conseguem disponibilizar conteúdos à escala global e mobilizar a participação empenhada de agentes que dispunham outrora de reduzida margem de intervenção no plano cultural, as Humanidades Digitais têm conseguido estabelecer novas pontes e quebrar preconceitos no que toca ao relacionamento entre o académico altamente especializado e o indivíduo que mesmo não sendo parte integrante da estrutura universitária manifesta um alto índice de curiosidade intelectual ou, através de pulsão autodidata, acumulou informação sobre determinados fenómenos. Ora, expressões como Crowd Science, Citizen Science ou Networked Science têm sido usadas para designar uma série de projetos que têm em comum a configuração participativa, a partir de número considerável de utilizadores e a partilha aberta de contributos em fases intermédias do processo de pesquisa. Em especial no terreno das Humanidades, várias experiências de crowdsourcing, no sentido de utilização de novos meios para tirar partido da sabedoria das multidões (wisdom of crowds, na linha da proposta de James Surowiecki), produziram resultados muito convincentes e deixam no ar a ideia de um forte potencial a explorar (Surowiecki, 2004). Entre todos, o caso mais conhecido (e ilustrativo da base de trabalho) é o da Wikipedia, mas há fenómenos como o Overmundo, website colaborativo sobre cultura brasileira, ${ }^{48}$ ou projetos, em versão ainda mais sofisticada, como o Hypermedia Berlin $^{49}$ e o Hypercities, ${ }^{50}$ lançados por Todd Presner, que disponibilizam software de fonte aberta para construir repositórios de informação, Transcribe

48 Overmundo (http://www.overmundo.com.br/). Para uma análise dos desafios trazidos por estes novos processos autorais através de redes eletrónicas, que pressupõem uma ampla partilha de informações, cf. Martins, 2014.

${ }^{49}$ Hypermedia Berlin (http://www.berlin.ucla.edu/).

${ }^{50}$ HyperCities - Thick Mapping in the Digital Humanities (http://www.hypercities.com/). 
Bentham ${ }^{51}$ ou Letters of $1916 .{ }^{52}$ No contexto português, um dos casos mais frutuosos e com pendor pioneiro neste âmbito é o projeto Portugal 14-18, desenvolvido pela Universidade Nova de Lisboa, que permitiu recolher material e memórias da Primeira Guerra Mundial. ${ }^{53}$ É de esperar, nos próximos anos, um crescimento muito significativo das tecnologias de colaboração com efeitos palpáveis em termos de conhecimento a que se pode aceder mais facilmente, disponível e pesquisável.

\section{Conclusão}

Somos confrontados, no momento presente, com questões que não podem ser resolvidas apenas com recurso à tecnologia e que requerem um contributo decisivo da cultura humanística. Por outro lado, algumas das novas questões que atravessam o nosso mundo tecnológico são, na realidade, velhas questões, ainda que com distinta formulação e outras implicações, que estão no centro das preocupações do ser humano há muito tempo. Nesse sentido, procurar reduzir a missão das Humanidades Digitais à mera aplicação de ferramentas e recursos digitais releva de uma atitude simplista, pois o modo como boa parte dos investigadores que se reconhecem como agentes (e seguramente também como construtores) deste campo estão empenhados em debater e testar novos modelos de compreensão da cultura e do mundo. Que essa dimensão especulativa e que as possibilidades de experimentação criativa tenham origem no terreno do digital e com forte apoio no conhecimento computacional não significa que se pretenda agora fazer o mesmo que antes já se fazia (ou muito próximo disso) apenas recorrendo a uma série de novos e mais sofisticados aparatos tecnológicos. Se o conjunto mais ou menos articulado de práticas a que têm recorrido todos os que gravitam em torno deste campo procura satisfazer necessidades de caráter utilitário, não se pode esquecer que dá também forma material a uma narrativa fundamental de autoidentidade.

Fazer valer a forte tradição de pensamento crítico, que aparece destacada no título deste ensaio e que sempre caraterizou a cultura de base humanística, é um objetivo que em nada desmerece a missão. Como por várias vezes se procurou demonstrar, quer sob ponto de vista das matrizes teóricas em que se apoia, por mais díspares que estas sejam, quer em termos metodológicos e de política de investigação, é inegável o potencial disruptivo que as Humanidades Digitais trouxeram ao espaço académico tradicional. Se a transformação profunda que se verificou a nível da condição contemporânea do saber é, em grande medida, o resultado da alteração dos meios tecnológicos de pro-

51 Transcribe Bentham - A Participatory Initiative (http://blogs.ucl.ac.uk/transcribebentham/).

${ }^{52}$ Letters of 1916: A Year in the Life (http://dh.tcd.ie/letters1916/).

53 Portugal 14-18 (http:/ / www.portugal1914.org/). 
dução, gestão e arquivamento do conhecimento, não deixa de refletir também os efeitos de um mundo globalizado, atravessado por múltiplas tensões (diversidade cultural; defesa da variedade linguística vs. assunção plena de uma língua franca), pelo que é nessa complexa rede que devemos situar o âmago da questão.

À semelhança do que sucede com a presença digital de pesquisa em língua espanhola na área das Humanidades, também no que toca a patrimónios de língua portuguesa se pode constatar um desempenho claramente inferior ao de outras tradições e culturas, nomeadamente de matriz anglo-saxónica. $\mathrm{Na}$ raiz do problema estão por certo condicionalismos exógenos, que ultrapassam as fronteiras do campo e sobre os quais nem sempre é fácil conseguir intervir, mas há reflexão e trabalho efetivo que deve ser realizado no plano institucional-académico na área geográfico-cultural transatlântica. Cultivar o sentido de comunidade com base numa língua de comunicação internacional com projeção global e património de outros patrimónios como é a língua portuguesa, através de redes e plataformas de comunicação, permitiria configurar uma área académica transnacional e cosmopolita, fortalecendo o desígnio de cooperação internacional, a aproximação entre os povos e a superação do estatuto de subalternidade que atualmente se verifica na cartografia global das Humanidades Digitais.

\section{Referências}

ALVES, Daniel (2014). "From "Humanities and Computing" to "Digital Humanities": Digital Humanities in Portugal with a focus on Historical Research." H-Soz-Kult. 24 de outubro. http://www.hsozkult.de/hfn/debate/id/diskussionen-2455.

BAILEY, Moya Z. (2011). "All the Digital Humanists Are White, All the Nerds Are Men, but Some of Us Are Brave." JDH: Journal of Digital Humanities, 1.1. 21 de setembro de 2015.

http://journalofdigitalhumanities.org/1-1/all-the-digital-humanists-arewhite-all-the-nerds-are-men-but-some-of-us-are-brave-by-moya-zbailey/.

BERRY, David M., ed. (2012). Understanding Digital Humanities. Basingstoke: Palgrave Macmillan.

BORGMAN, Christine L. (2015a). Big Data, Little Data, No Data: Scholarship in the Networked World. Cambridge, MA: MIT Press.

et. al. (2015b). "Knowledge Infrastructures in Science: Data, Diversity, and Digital Libraries." International Journal on Digital Libraries 16.47.

BURDICK, Anne, et. al. (2012). Digital_Humanities. Cambridge, Mass: The MIT Press. Open Access Edition. 21 de setembro de 2015. https://mitpress.mit.edu/sites/default/files/titles/content/9780262018 470_Open_Access_Edition.pdf. 
CRANE, Gregory (2006). "What Do You Do with a Million Books?.” D-Lib Magazine 12.3. 21 de setembro de 2015. http://www.dlib.org/dlib/march06/crane/03crane.html.

, Brent Seales e Melissa Terras (2009). "Cyberinfrastructure for Classical Philology." Digital Humanities Quarterly 3.1. 21 de setembro de 2015.

http://www.digitalhumanities.org/dhq/vol/003/1/000023/000023.html

DACOS, Marin (2013). "La stratégie du Sauna finlandais: Les frontières de Digital Humanities. Essai de Géographie politique d'une communauté scientifique." 21 de setembro de 2015. hal-00866107.

DEEGAN, Marilyn e Sutherland, Kathryn, eds (2009). Transferred Illusions. Digital technology and the forms of print. Farnham: Ashgate.

ESCANDELL MONTIEL, Daniel (2012). Narrativa digital hispana: el blog como espacio de creación literaria a comienzos del siglo XXI. Salamanca: Universidad de Salamanca.

FIORMONTE, Domenico (2012). "Towards a Cultural Critique of Digital Humanities." Historical Social Research/Historische Sozialforschung 37. http://www.cceh.uni-koeln.de/files/Fiormonte_0.pdf.

(2014). "Digital Humanities from a global perspective." Laboratorio dell'ISPF XI. 21 de setembro de 2015. http://www.ispflab.cnr.it/2014_203.pdf.

FRYE, Northrop (2006). The Secular Scripture and Other Writings on Critical Theory, 1976-1991. Eds. Joseph Adamson and Jean Wilson. Toronto: University of Toronto Press.

GALINA, Isabel (2013). "Is There Anybody Out There? Building a Global Digital Humanities Community." Humanidades Digitales. 21 de setembro de 2015. http://humanidadesdigitales.net/blog/2013/07/19/is-thereanybody-out-there-building-a-global-digital-humanities-community/.

Nova ed. revista e aumentada: (2014). "Geographical and Linguistic Diversity in the Digital Humanities." Literary and Linguistic Computing 29.3: 307-316.

GIBBS, Frederick W. e Daniel J. Cohen (2011). "A Conversation with Data: Prospecting Victorian Words and Ideas." Victorian Studies 54.1: 69-77. 21 de setembro de 2015. http://www.dancohen.org/2012/05/30/aconversation-with-data-prospecting-victorian-words-and-ideas/.

GOLD, M. K., ed. (2012). Debates in the Digital Humanities. Minneapolis: University of Minnesota Press. 21 de setembro de 2015. http://dhdebates.gc.cuny.edu/.

GOLUMBIA, David (2013). "Postcolonial Studies, Digital Humanities, and the Politics of Language." 31 de maio. 21 de setembro de 2015. http://www.uncomputing.org/?p=241.

GUMBRECHT, Hans Ulrich (2004). Production of Presence: What Meaning Cannot Convey. Stanford: Stanford UP. 
HONN, Josh (2013). "Never Neutral: Critical Approaches to Digital Tools \& Culture in the Humanities." 17 de outubro. 21 de setembro de 2015. http:/ / sites.northwestern.edu/jch629/2013/10/17/never-neutralcritical-approaches-to-digital-tools-culture-in-the-humanities/.

KIRSCHENBAUM, Matthew (2014). "What is 'Digital Humanities,' and Why Are They Saying Such Terrible Things about It?.” differences 25.1: 4663. 21 de setembro de 2015. DOI 10.1215/10407391-2419997.

KOH, Adeline, e Roopika Risam (2013). "Open Thread: The Digital Humanities as a Historical "Refuge" from Race/Class/Gender/Sexuality/ Disability?.” 21 de setembro de 2015. http:/ /dhpoco.org/blog/2013/05/10/open-thread-the-digital-humanitiesas-a-historical-refuge-from-raceclassgendersexualitydisability/.

$\mathrm{KOH}$, Adeline (2014). "Niceness, Building, and Opening the Genealogy of the Digital Humanities: Beyond the Social Contract of Humanities Computing." differences 25.1: 93-106.

JOCKERS, Matthew (2013). Macroanalysis: Digital Methods and Literary History, Urbana: University of Illinois.

LAFUENTE, Antonio, Andoni Alonso e Joaquín Rodríguez (2013). ¡Todos sabios! Ciencia ciudadana y conocimiento expandido. Madrid: Cátedra.

LIU, Alan (2011). "The State of the Digital Humanities: A Report and a Critique." Arts and Humanities in Higher Education 11.1-2: 8-41.

(2012). "Where is Cultural Criticism in the Digital Humanities?." Debates in the Digital Humanities. Ed. M. Gold. Minneapolis, University of Minnesota Press. 490-509.

LODGE, David (1984). Small World: An Academic Romance. London: Secker \& Warburg.

LOTHIAN, Alexis e Phillips, Amanda (2013). "Can Digital Humanities Mean Transformative Critique?.” Journal of e-Media Studies 3.1. Dartmouth College. 21 de setembro de 2015. http://journals.dartmouth.edu/cgibin/WebObjects/Journals.woa/1/xmlpage/4/article/425.

MARTELL, Luke (2014). "The Slow University: Inequality, Power and Alternatives." Forum Qualitative Sozialforschung / Forum: Qualitative Social Research 15.3. Art. 10. 21 de setembro de 2015. http://nbnresolving.de/urn:nbn:de:0114-fqs1403102.

MAYER-SCHÖNBERGER, Viktor e Kenneth Cukier (2013). Big Data: A Revolution That Will Transform How We Live, Work and Think. London: John Murray.

MARTINS, Beatriz Cintra (2014). Autoria em rede. Os novos processos autorais através das redes eletrônicas. Rio de Janeiro: Mauad Editora.

MCCARTY, Willard (2004). "Modeling: A Study in Words and Meanings." $A$ Companion to Digital Humanities. Eds. Susan Schreibman, Ray Siemens, John Unsworth. Oxford: Blackwwell. 254-270. 21 de setembro de 2015. http://www.digitalhumanities.org/companion/. 
(2012). "The Residue of Uniqueness." 21 de setembro de 2015. http://www.cceh.uni-koeln.de/files/McCarty.pdf.

MICHEL, Jean-Baptiste, et al. (2011). "Quantitative Analysis of Culture Using Millions of Digitized Books." Science 33.6014: 176-182. 20 de setembro de 2015. https://dash.harvard.edu/handle/1/8899722.

MORETTI, Franco (2005). Graphs, Maps, Trees: Abstract Models for a Literary History. London and New York: Verso.

(2013). Distant Reading. London: Verso.

ORTEGA, E. e Gutiérrez, S. (2012). MapaHD. 20 de setembro de 2015. http://mapahd.org/el-mapa/.

PAIXÃO DE SOUSA, Maria Clara (2011). "As Humanidades e as Tecnologias Digitais: Uma Provocação Inicial. Humanidades Digitais.” 20 de setembro de 2015.

http://humanidadesdigitais.org/2011/07/07/por-que-humanidadesdigitais/.

PEREIRA, Paulo Silva (2015). "Barroco Digital: Remediação, Edição Textual e Arquivo." Revista Colóquio/Letras 188, jan: 20-32.

PORTELA, Manuel (2013a). Scripting Reading Motions. The Codex and The Computer as Self-Reflexive Machines. Cambridge, Mass.: MIT Press.

(2013b). "Nenhum Problema Tem Solução: Um Arquivo Digital do Livro do Desassossego." MatLit 1.1: 9-33. 20 de setembro de 2015. http://iduc.uc.pt/index.php/matlit/article/view/1618.

PRESNER, Todd (2012). "Critical Theory and the Mangle of Digital Humanities." 20 de setembro de 2015. http://www.toddpresner.com/wpcontent/uploads/2012/09/Presner_2012_DH_FINAL.pdf.

RAMSAY, Stephen (2011a). "Who's In and Who's Out." 20 de setmbro de 2015. http://stephenramsay.us/text/2011/01/08/whos-in-and-whosout/.

(2011b). “On Building." 20 de setembro de 2015. http://stephenramsay.us/text/2011/01/11/on-building/.

RÖHRS, H., ed. (1987). Tradition and Reform of the University under an International Perspective. New York: Peter Lang.

ROJAS CASTRO, Antonio (2013). "El mapa y el territorio. Una aproximación histórico-bibliográfica a la emergencia de las Humanidades Digitales en España." Caracteres. Estudios culturales y críticos de la esfera digital. 20 de setembro de 2015.

http://revistacaracteres.net/revista/vol2n2noviembre2013/el-mapa-y-elterritorio/.

ROMERO FRÍAS, Esteban e María Sánchez González, eds. (2014). “Ciencias Sociales y Humanidades Digitales. Técnicas, herramientas y experiencias de e-Research e investigación en colaboración." CAC: Cuadernos Artesanos de Comunicación 61. 20 de setembro de 2015.

http:/ / www.cuadernosartesanos.org/2014/cac61.pdf. 
SANZ, Amelia (2013). "Digital Humanities or Hypercolonial Studies?” RICT. Responsible Innovation. 21 de setembro de 2015. http://responsibleinnovation.org.uk/resource-detail/1249.

SCHNAPP, Jeffrey e Todd Presner (2009). Digital Humanities Manifesto 2.0. 21 de setembro de 2015.

http://www.humanitiesblast.com/manifesto/Manifesto_V2.pdf.

SCHREIBMAN, Susan, Ray Siemens e John Unsworth, eds. (2004). A Companion to Digital Humanities. Oxford: Blackwell. 21 de setembro de 2015. http://www.digitalhumanities.org/companion/.

SUROWIECKI, James (2004). The Wisdom of Crowds: Why the Many Are Smarter Than the Few and How Collective Wisdom Shapes Business, Economies, Societies and Nations. Little: Brown.

TERRAS, Melissa (2013). "On Changing the Rules of Digital Humanities from the Inside." Melissa Terra's Blog: Adventures in DH and Digital Cultural Heritage, Plus Some Musings on Academia. 27 de maio. 21 de setembro de 2015. http://melissaterras.blogspot.co.uk/2013/05/on-changing-rulesof-digital-humanities.html.

UNSWORTH, John (2004). "Forms of Attention: Digital Humanities Beyond Representation." A paper delivered at The Face of Text: ComputerAssisted Text Analysis in the Humanities, the third conference of the Canadian Symposium on Text Analysis (CaSTA). McMaster University. 19-21 de novembro. 21 de setembro de 2015.

http://people.brandeis.edu/ unsworth/FOA/.

(C) 2015 Paulo Silva Pereira.

Licensed under the Creative Commons Attribution-Noncommercial-

No Derivative Works 4.0 International (CC BY-NC-ND 4.0). 